\title{
Thiazolidinediones increase the wingless-type MMTV integration site family (WNT) inhibitor Dickkopf-1 in adipocytes: a link with osteogenesis
}

\author{
B. Gustafson • B. Eliasson • U. Smith
}

Received: 24 July 2009 / Accepted: 30 October 2009 / Published online: 27 November 2009

(C) Springer-Verlag 2009

\begin{abstract}
Aims/hypothesis Dickkopf-1 (DKK1) is a secreted inhibitor of canonical wingless-type MMTV integration site family (WNT) signalling; the key pathway for cell fate and development. Inhibition of WNT signalling by DKK1 in precursor cells promotes adipogenesis and inhibits osteogenesis. Previous studies have shown that treatment of type 2 diabetic patients with thiazolidinediones (TZDs) reduces bone density and increases risk of bone fractures, while body fat is increased.

Methods We examined the effect of TZDs on secretion and DKK1 levels in pre-adipocytes and mature adipose cells and also measured circulating DKK1 levels in 11 patients with type 2 diabetes before and after treatment with the TZD rosiglitazone for 90 days.

Results TZDs added in vitro rapidly increased DKK1 protein levels and secretion in both fully differentiated adipose cells and pre-adipocytes undergoing differentiation. In parallel, $\beta$-catenin levels, a marker of canonical WNT signalling, were reduced. Serum levels of DKK1 were also increased in several of the patients with type 2 diabetes after treatment with rosiglitazone for 90 days.

Conclusions/interpretation These results provide a novel mechanism whereby peroxisome proliferator-activated receptor- $\gamma$ activation can terminate WNT signalling and promote adipogenesis. Furthermore, they provide an explanation for why TZD treatment can lead to reduced bone
\end{abstract}

B. Gustafson · B. Eliasson · U. Smith $(\bowtie)$

The Lundberg Laboratory for Diabetes Research,

Center of Excellence for Metabolic and Cardiovascular Research,

Department of Molecular and Clinical Medicine,

Sahlgrenska University Hospital,

Blå Stråket 3,

41345 Gothenburg, Sweden

e-mail: ulf.smith@medic.gu.se formation and increased risk of fractures, since inhibited WNT signalling in progenitor cells promotes adipogenesis while osteogenesis is reduced.

Keywords Adipocytes · Adipogenesis · Dickkopf-1 · Osteogenesis $\cdot$ PPAR $\gamma \cdot$ Thiazolidinediones $\cdot$ WNT signalling

$\begin{array}{ll}\text { Abbreviations } \\ \text { DKK1 } & \text { Dickkopf-1 } \\ \text { ERK1 } & \begin{array}{l}\text { Extracellular signal-regulated kinase 1 } \\ \text { Low-density lipoprotein receptor-related } \\ \text { LRP5/6 }\end{array} \\ \text { protein 5-6 } \\ \text { PPAR } \gamma & \begin{array}{l}\text { Peroxisome proliferator-activated receptor- } \gamma \\ \text { TMB }\end{array} \\ \text { TZD } & \text { Thiazolidinedione } \\ \text { WNT } & \text { Wingless-type MMTV integration site family }\end{array}$

\section{Introduction}

Thiazolidinediones (TZDs) are peroxisome proliferatoractivated receptor- $\gamma$ (PPAR $\gamma$ ) agonists that are commonly used to treat type 2 diabetes and insulin resistance. A key mechanism of action is to increase the differentiation of (pre-) adipocytes, thus increasing adiponectin secretion and lipid storage. In addition, they exert anti-inflammatory effects through the transrepression of proinflammatory genes (for review see Yang and Smith [1]).

A recent concern with these agents is the demonstration of an annual, about 1\%/year, bone loss in postmenopausal women combined with an increased risk of bone fractures [2-4]. The mechanism(s) for this is not clear but several studies have shown that TZDs promote the differentiation of mesenchymal stem cells towards the adipose lineage rather than into osteoblastogenesis $[5,6]$. This, in turn, 
leads to fewer osteoblasts being available for differentiation, while adipose cell formation, including in the bone marrow, would be favoured.

The major pathway for regulating cell fate and committing mesenchymal stem cells to osteoblasts is the canonical wingless-type MMTV integration site family (WNT) signalling pathway [7]. Activation of this pathway suppresses adipogenesis and promotes osteoblast formation. The Kremen/low-density lipoprotein receptor-related protein 5-6 (LRP5/6) ligand Dickkopf-1 (DKK1) is a soluble inhibitor of WNT signalling and it has also been identified as a key regulator of osteoblastogenesis. Recent studies have shown that DKK1 levels are increased by TNF- $\alpha$ in osteoblasts, thus preventing their differentiation and linking it to rheumatoid arthritis with impaired bone formation and increased bone destruction [8]. Similarly, myeloma cells secrete DKK1, leading to osteolytic bone destruction, which can be inhibited by anti-DKK1 antibodies in animal experiments [9]. Although mainly exerting its effect at the cellular level with intercellular cross-talk, secreted DKK1 enters the bloodstream and elevated levels have been reported in patients with rheumatoid arthritis and multiple myeloma $[8,10]$. It is also probable that DKK1 can play an important role in the bone destruction seen in other cancer forms, although this needs to be further explored.

As an inhibitor of WNT signalling, DKK1 can be expected to promote adipogenesis, since active WNT signalling prevents both precursor cells from entering the adipose lineage and the normal differentiation of committed pre-adipocytes [11-13]. DKK1 binds to both Kremen and the LRP receptor, thus preventing WNT from binding and activating the WNT signal [14].

We here examined DKK1 protein levels in fully differentiated adipose cells as well as in pre-adipocytes undergoing differentiation with or without the addition of a TZD. Under both conditions, TZDs increased the protein levels of DKK1 and reduced levels of $\beta$-catenin, the intracellular mediator of WNT signalling. We also measured DKK1 levels in the blood of diabetic patients before and after the initiation of TZD therapy for 3 months. Intriguingly, serum DKK levels were also increased in several patients. Thus, our results show for the first time that TZDs can antagonise WNT signalling through induction of DKK1. This, in turn, favours adipogenesis and adipose cell differentiation at the expense of osteogenesis. DKK1 activation is probably an important early mechanism whereby PPAR $\gamma$ can terminate the WNT signal and promote adipogenesis.

\section{Methods}

Materials All chemicals were of high quality and obtained from commercial sources. Immunoblots and ELISA were performed with the following antibodies and reagents: $\beta$-catenin (Transduction Laboratories, BD Biosciences, San Jose, CA, USA); extracellular signal-regulated kinase (ERK) 1 (Upstate [01-182], Millipore, Stockholm, Sweden); and DKK1, LRP6/Fc (Fc region of human immunoglobulin G linked to human LRP6) chimera, human (h) DKK1 and recombinant hDKK1 (all R\&D Systems [monoclonal antibody 1765, 1505-LR, BAF1096, 1096DK], Abingdon, UK). Streptavidin-peroxidase and 3,3',5,5'tetramethylbenzidine (TMB) were from Sigma-Aldrich, Stockholm, Sweden.

Study population We recruited 12 type 2 diabetic patients to this study via a newspaper advertisement. The regional ethical review board approved the study, which was carried out in accordance with the Principles of the Declaration of Helsinki. Informed consent was obtained from all patients.

The patients recruited to this explorative open trial were non-symptomatic type 2 diabetic patients (one woman, 11 men) on stable treatment with diet or oral hypoglycaemic agents. Rosiglitazone $8 \mathrm{mg}$ once per day was added to the treatment regimen in accordance with the approved label. Other clinically significant diseases or symptoms, such as ischaemic heart disease, thyroid disease or $\mathrm{HbA}_{1 \mathrm{c}}>$ $10 \%$ were exclusion criteria. All patients were nonsmokers. The patients were encouraged not to change their lifestyle habits during the study. One lean, previously untreated patient (BMI $20 \mathrm{~kg} / \mathrm{m}^{2}$ ) had previously been diagnosed as having type 2 diabetes on clinical grounds but was excluded because of low serum insulin and C-peptide levels and rapidly deteriorating glycaemic control. Thus, the results are based on the 11 patients who concluded the 90 day treatment period.

Before the study, three patients were on diet treatment, three on sulfonylurea, two on repaglinide, two on metformin and one on sulfonylurea and metformin combined. Seven patients were treated with antihypertensive agents and four with statins for hyperlipidaemia. Fasting blood samples for DKK1 measurements were collected before treatment and 90 days after the initiation of rosiglitazone treatment.

Cell culture conditions Mouse 3T3-L1 pre-adipocytes were cultured in DMEM supplemented with 10\% (vol./vol.) FBS, $2 \mathrm{mmol} / \mathrm{l}$ glutamine and antibiotics, as previously described [12]. After 2 days of confluence, the cells were treated with a differentiation cocktail consisting of $0.5 \mathrm{mmol} / 1$ methyl-isobutylxanthine, $1 \mu \mathrm{mol} / 1$ dexamethasone and $865 \mathrm{nmol} / 1$ insulin for $48 \mathrm{~h}$, followed by insulin alone for an additional $48 \mathrm{~h}$. Pioglitazone $(1 \mu \mathrm{mol} / \mathrm{l})$ was added to the differentiation cocktail and to the cell culture media during the 8 days of differentiation. In a separate 
study, rosiglitazone or pioglitazone $(1 \mu \mathrm{mol} / \mathrm{l})$ was added to the cell culture media for the indicated time after 8 days of differentiation. These cells had then first undergone full differentiation to adipose cells. Thus, two different TZDs were tested as well as cells undergoing differentiation (preadipocytes) or having already become fully differentiated adipose cells.

Whole cell extracts and immunoblotting 3T3-L1 adipocytes were rinsed with ice-cold PBS and lysed in lysis buffer as previously reported [12]. The insoluble material was sedimented by centrifugation at $20,000 \mathrm{~g}$ for $10 \mathrm{~min}$ at $4^{\circ} \mathrm{C}$ and the supernatant fractions were collected and frozen at $-80^{\circ} \mathrm{C}$. Protein content was determined using a BCA Protein Assay kit (Pierce, Rockford, IL, USA). Forty micrograms of whole cell extracts was used for electrophoresis through 10\% SDSpolyacrylamide gels (Lonza Group, Basel, Switzerland). For detection of secreted DKK1, $120 \mu \mathrm{l}$ of cell culture medium was used for electrophoresis through large 10\% SDSpolyacrylamide gels made in the laboratory. The proteins were transferred to BA85 nitrocellulose membranes (Schleicher \& Schuell, VWR, Stockholm, Sweden). The membranes were blocked for $1 \mathrm{~h}$ with $5 \%$ (wt./vol.) dried milk in TRIS-saline (T/S) buffer $(10 \mathrm{mmol} / \mathrm{l}$ TRIS-base, $0.154 \mathrm{~mol} / 1 \mathrm{NaCl}$ and $1 \%$ (vol./vol.) Tween 20). After washing in $\mathrm{T} / \mathrm{S}$ buffer, the membranes were incubated with primary antibodies overnight. For detection, the membranes were incubated with secondary antibody for $1 \mathrm{~h}$ and the bands were visualised with an Immune-Star HRP Chemiluminescence Kit (Bio-Rad Laboratories, Hercules, CA, USA) for quantification with a ChemiDoc XRS System (Bio-Rad Laboratories).

ELISA assay Frozen serum samples were analysed with ELISAs in 96 well plates [15]. The plates were coated with $1 \mu \mathrm{g} / \mathrm{ml}$ human LRP6/Fc chimera for $16 \mathrm{~h}$ at $4^{\circ} \mathrm{C}$. All the following steps were performed at room temperature. The plates were washed three times with PBS containing $0.05 \%$ (vol./vol.) Tween 20 (PBST) and blocked for $1 \mathrm{~h}$ in PBS with 4\% (wt./vol.) BSA and $0.1 \%$ (vol./vol.) Tween 20 . Patient samples were diluted $1: 4$, and $100 \mu 1$ of the diluted samples were loaded into each well. The plates were incubated for $2 \mathrm{~h}$ at room temperature and then washed three times with PBST. To each well, $100 \mu \mathrm{l}$ biotin-labelled anti-human DKK1 was added and the plates were incubated for $2 \mathrm{~h}$. For detection, $100 \mu \mathrm{l}$ streptavidin-peroxidase polymer was added for $30 \mathrm{~min}$. After washing, development of the reaction was done by addition of $100 \mu \mathrm{TMB}$ substrate. The reaction was stopped with $50 \mu 11 \mathrm{~mol} / 1 \mathrm{H}_{2} \mathrm{SO}_{4}$ and the extinction was measured using a VERSAmax microplate reader (Molecular Devices, Sunnyvale, CA, USA) at 540 and $450 \mathrm{~nm}$. Evaluation was performed with SOFTmax PRO (Molecular Devices).
Real-time PCR (RT-PCR) Total RNA was isolated from the cells with RNeasy (Qiagen, Hilden, Germany). Quantitative RT-PCR was performed with the TaqMan system (Applied Biosystems, Foster City, CA, USA). Gene-specific primers and probes were designed using Primer Express software (Applied Biosystems) and the sequences used are available on request. The RT-PCR reaction was performed essentially as described before [12]. 18S ribosomal RNA was used as endogenous control. Analyses were performed in duplicates and all experiments were repeated at least three times.

Statistical analyses Student's $t$ test was used to compare protein samples from untreated and TZD-treated 3T3-L1 cells. Paired $t$ tests were used for comparison of patients treated or not with rosiglitazone. $p<0.05$ was considered statistically significant.

\section{Results}

When 3T3-L1 pre-adipocytes undergo normal differentiation to adipose cells, DKK1 is induced and, thus, contributes to the termination of the inhibitory WNT signals (Fig. 1a). Addition of pioglitazone further promoted the induction of DKK1 (Fig. 1a) while $\beta$-catenin, the indicator of canonical WNT signalling, were reduced (Fig. 1b). The data shown in Fig. 1 were repeated six times with similar results. DKK1 protein levels were significantly increased by TZD at both day $4(+31 \%, p=0.05)$ and day $8(+33 \%$,

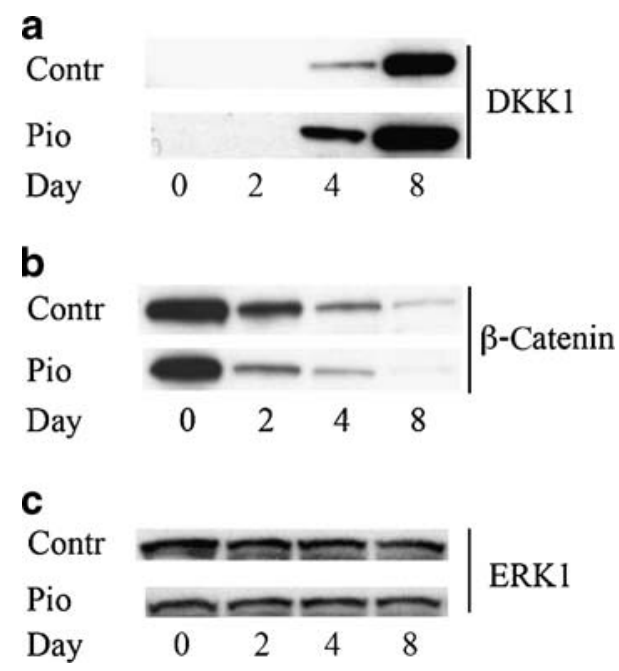

Fig. 1 TZD increases DKK1 protein levels during differentiation of 3T3-L1 pre-adipocytes. Induction of DKK1 can be identified between days 2 and 4 of differentiation (Contr) and addition of $1 \mu \mathrm{mol} / 1$ pioglitazone (Pio) to the differentiation cocktail enhances DKK1 protein levels (a). This is also associated with reduced $\beta$-catenin levels (b). One representative blot of six experiments is shown. ERK1 was used as loading control since the protein level of ERK1 is unchanged during differentiation $(\mathbf{c})$ 
Fig. 2 In mature adipocytes, $1 \mu \mathrm{mol} / \mathrm{l}$ rosiglitazone (Rosi) (a) or pioglitazone (Pio) (b) increases protein levels of DKK1 after 8-48 h compared with controls (Contr). ERK1 was used as a loading control (d). The rosiglitazone data were normalised to the protein level in control samples for each time point (c). Data are presented as means \pm SEM $(n=3) .{ }^{*} p<0.05$, $* * * p<0.001$

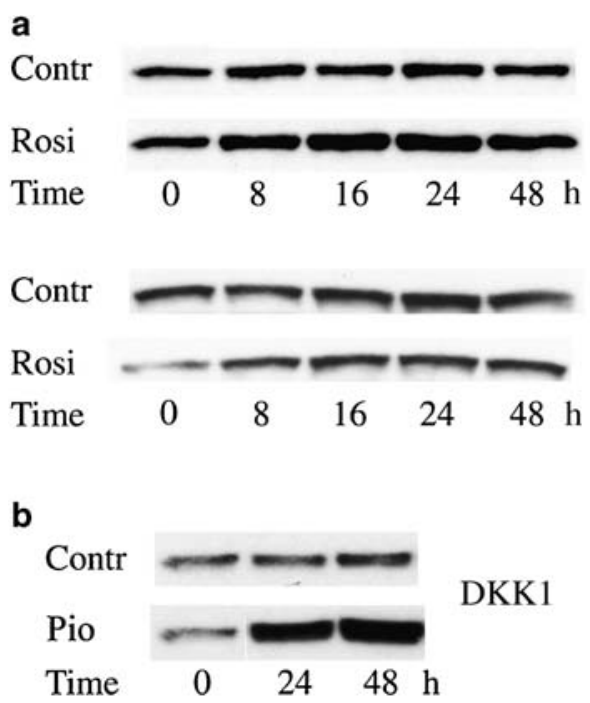

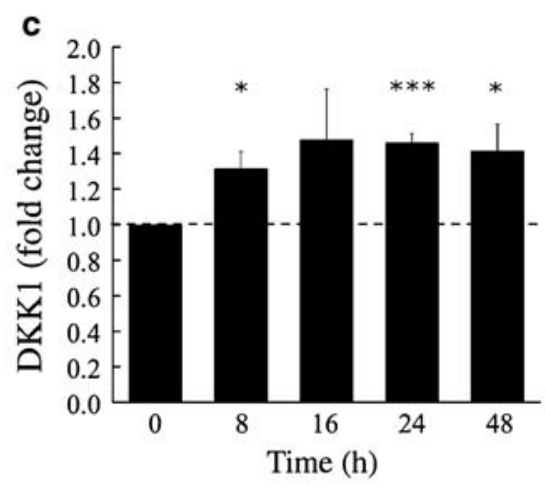

d

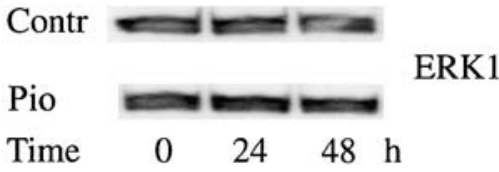

$p=0.02$ ) compared with the control cells. Similarly, $\beta$-catenin levels were significantly reduced in the presence of pioglitazone at both day $4(-33 \%, p=0.004)$ and day $8(-16 \%, p=0.033)$. These results suggest that induction of DKK1 is an important mechanism for the termination of WNT signalling in pre-adipocytes and that this is mediated through PPAR $\gamma$ activation. As expected, TZD addition also promoted the induction of specific adipocyte markers such as aP2, adiponectin and GLUT4 (data not shown).

We then tested if TZDs could also induce DKK1 in differentiated adipose cells, thus contributing to maintaining the cells in a fully differentiated state. Interestingly, differentiated adipose cells also showed increased DKK1 levels when either rosiglitazone or pioglitazone (Fig. 2a) was added and this was seen after $8 \mathrm{~h}$. Thus, DKK1 is a rapidly inducible protein by PPAR $\gamma$ activation in both preadipocytes and fully differentiated adipose cells. Furthermore, DKK1 is a secreted protein and a TZD also enhanced the secretion of DKK1 by the adipocytes to the ambient medium (Fig. 3). Unexpectedly, TZDs did not significantly increase $D K K 1$ mRNA expression in differentiated cells (data not shown), indicating that PPAR $\gamma$ activation stabilised the protein rather than increased the transcription, but the mechanism for this is currently unclear.

Although the WNT signalling pathway is mainly regulated locally through cross-talk at the cellular level, we also analysed circulating DKK1 levels in 11 type 2 diabetic individuals before and after the addition of rosiglitazone $8 \mathrm{mg}$ once per day for 3 months. As shown

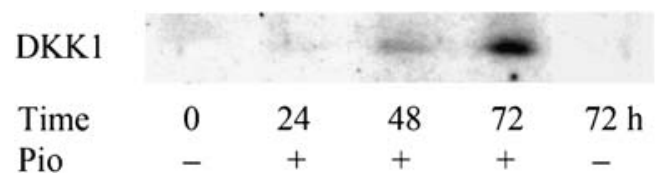

Fig. 3 The presence of pioglitazone (Pio) increases the secretion of DKK1 into the culture medium by fully differentiated adipose cells in Fig. 4, with the exception of one individual, there was a marked (five out of 11) or a slight (four out of 11) increase in the circulating DKK1 levels ( $p=0.07, n=11)$. (Excluding the one individual with a decrease, $n=10, p=0.02$.)

\section{Discussion}

Taken together, these results show a novel way through which TZDs can promote adipogenesis and terminate WNT signalling. Although TZDs improve adipocyte differentiation in general, the finding that DKK1 levels were also increased in fully differentiated cells implicates a direct effect of PPAR $\gamma$

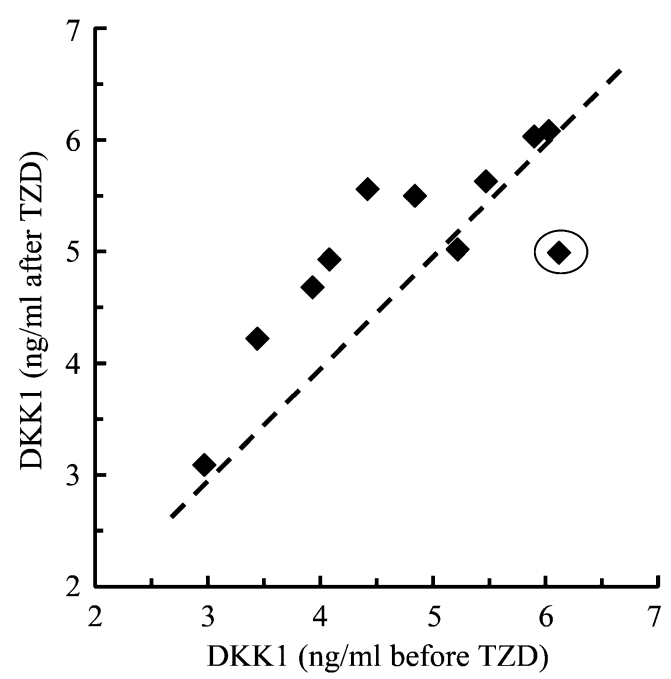

Fig. 4 Circulating DKK1 protein levels are increased in patients treated with a TZD. Eleven non-symptomatic type 2 diabetic patients were treated with $8 \mathrm{mg}$ rosiglitazone once per day for 90 days and serum was taken before and after treatment for DKK1 analysis. With the exception of one patient (circled), most patients increased DKK1 serum levels $(p=0.07)$. (Exclusion of the indicated patient; $n=10$, $p=0.02$ ) 
activation on this protein. However, the mechanism for this is currently unclear. Furthermore, increasing DKK1 allows TZDs to antagonistically regulate the fate of osteogenic and adipose cell precursors, thereby promoting adipose cell differentiation at the expense of osteogenesis. In addition to increasing circulating levels of DKK1 from the peripheral adipose depots, one can envisage that the adipose cells in the bone marrow, which increase in number with age [16], will produce high local DKK1 levels following TZD treatment. This, in turn, will prevent the transition of the stem cells to osteoblasts while favouring adipogenesis. Previous studies have indeed shown that TZDs promote stem cell differentiation to adipocytes [5, 6], although the mechanism(s) for this has so far been unknown. Although we only tested one TZD in the in vivo study we saw the induction of DKK1 in vitro with both rosiglitazone and pioglitazone, making it likely that this effect is intrinsic to PPAR $\gamma$ activation. However, testing other PPAR $\gamma$ agonists for this effect may allow the development of agents that have a selective profile and lack effects on bone formation.

In addition to identifying a novel mechanism whereby PPAR $\gamma$ activation can promote adipogenesis, these results also raise a concern for the use of TZDs in conditions where increased secretion of DKK1 may influence the clinical course, such as in multiple myeloma. We have been unable to find any clinical analyses of this in the literature, but the present findings strongly indicate the need for such investigations.

Acknowledgements We wish to thank the Swedish Research Council, the Swedish Diabetes Association, the Novo Nordisk Foundation, the Swedish Foundation for Strategic Research and the Torsten and Ragnar Söderberg Foundation for financial support. We also acknowledge an unrestricted research grant from GlaxoSmithKline, Sweden.

Duality of interest The authors declare that there is no duality of interest associated with this manuscript.

\section{References}

1. Yang X, Smith U (2007) Adipose tissue distribution and risk of metabolic disease: does thiazolidinedione-induced adipose tissue redistribution provide a clue to the answer? Diabetologia 50:1127-1139
2. Grey A, Bolland M, Gamble G et al (2007) The peroxisome proliferator-activated receptor-gamma agonist rosiglitazone decreases bone formation and bone mineral density in healthy postmenopausal women: a randomized, controlled trial. J Clin Endocrinol Metab 92:1305-1310

3. Kahn SE, Zinman B, Lachin JM et al (2008) Rosiglitazoneassociated fractures in type 2 diabetes: an analysis from A Diabetes Outcome Progression Trial (ADOPT). Diabetes Care 31:845-851

4. Schwartz AV, Sellmeyer DE, Vittinghoff E et al (2006) Thiazolidinedione use and bone loss in older diabetic adults. J Clin Endocrinol Metab 91:3349-3354

5. Benvenuti S, Cellai I, Luciani P et al (2007) Rosiglitazone stimulates adipogenesis and decreases osteoblastogenesis in human mesenchymal stem cells. J Endocrinol Invest 30:RC26-RC30

6. Shockley KR, Lazarenko OP, Czernik PJ, Rosen CJ, Churchill GA, Lecka-Czernik B (2009) PPARgamma2 nuclear receptor controls multiple regulatory pathways of osteoblast differentiation from marrow mesenchymal stem cells. J Cell Biochem 106:232246

7. Gregory CA, Singh H, Perry AS, Prockop DJ (2003) The Wnt signaling inhibitor dickkopf- 1 is required for reentry into the cell cycle of human adult stem cells from bone marrow. J Biol Chem 278:28067-28078

8. Diarra D, Stolina M, Polzer K et al (2007) Dickkopf-1 is a master regulator of joint remodeling. Nat Med 13:156-163

9. Yaccoby S, Ling W, Zhan F, Walker R, Barlogie B, Shaughnessy JD Jr (2007) Antibody-based inhibition of DKK1 suppresses tumor-induced bone resorption and multiple myeloma growth in vivo. Blood 109:2106-2111

10. Politou MC, Heath DJ, Rahemtulla A et al (2006) Serum concentrations of Dickkopf-1 protein are increased in patients with multiple myeloma and reduced after autologous stem cell transplantation. Int J Cancer 119:1728-1731

11. Kennell JA, MacDougald OA (2005) Wnt signaling inhibits adipogenesis through beta-catenin-dependent and -independent mechanisms. J Biol Chem 280:24004-24010

12. Gustafson B, Smith U (2006) Cytokines promote Wnt signaling and inflammation and impair the normal differentiation and lipid accumulation in 3T3-L1 preadipocytes. J Biol Chem 281:95079516

13. Isakson P, Hammarstedt A, Gustafson B, Smith U (2009) Impaired preadipocyte differentiation in human abdominal obesity: role of Wnt, tumor necrosis factor-alpha, and inflammation. Diabetes 58:1550-1557

14. Mao B, Wu W, Li Y et al (2001) LDL-receptor-related protein 6 is a receptor for Dickkopf proteins. Nature 411:321-325

15. Lee N, Smolarz AJ, Olson S et al (2007) A potential role for Dkk-1 in the pathogenesis of osteosarcoma predicts novel diagnostic and treatment strategies. Br J Cancer 97:1552-1559

16. Gimble JM, Zvonic S, Floyd ZE, Kassem M, Nuttall ME (2006) Playing with bone and fat. J Cell Biochem 98:251-266 